\title{
Preparation of PMMA/PGMA Blend to Apply for Metallographic Cross Section of Printed Circuit Board
}

\section{Xue-Mei HE ${ }^{1}$, Tao SHEN ${ }^{1}$, Shou-Xu WANG ${ }^{1}$, Yuan-Ming CHEN ${ }^{1, *}$, Huai-Wu Zhang ${ }^{1}$, Li-Jun GONG ${ }^{2}$, Bei CHEN ${ }^{2}$ and Wei HE HE $^{1,3}$}

${ }^{1}$ State Key laboratory of electronic thin films and integrated devices, University of Electronic Science and Technology of China, Chengdu 610054, P. R. China

${ }^{2}$ Research and development department, Guangzhou Fastprint Circuit Tech Co., Ltd, Guangzhou, 510663, China

${ }^{3}$ Research and development department, Guangdong Guanghua Sci-Tech Co., Ltd., Shantou 515000, China

*ymchen@uestc.edu.cn

Keywords: PMMA/PGMA blend, MMA, GMA, PCB, Metallographic cross section.

\begin{abstract}
Poly (methyl methacrylate)/poly (glycidyl methacrylate) (PMMA/PGMA) blend was prepared with the reaction of PMMA, glycidyl methacrylate (GMA) and methyl methacrylate (MMA) at room temperature. Effects of the proportions of MMA and GMA were investigated to analyze the performance of PMMA/PGMA blend. When the weight fraction of GMA in GMA/MMA was 0.25, PMMA/PGMA blend exhibited good performance as curing time of 29 min, volume shrinkage of $9.83 \%$, bending strength of $50 \mathrm{MPa}$, tensile strength of $31 \mathrm{MPa}$ and bonding strength of $1.52 \mathrm{MPa}$. PMMA/PGMA blend could well cover the cross section of printed circuit board.
\end{abstract}

\section{Introduction}

Printed circuit board (PCB) is an indispensable module for signal transmission of electronic products .PCB quality at each process should be monitored to finally obtain reliable PCB structure due to the limitation of its complex manufacture processes [1].

Metallographic cross section of PCB is a useful method to directly observe the inside structure of PCB to find out the defects of delamination, skip plating, undercut, conductive anodic filament and copper migration etc [2].However, the fixation of PCB section is hard to be completed to avoid the structure damage after cutting a local segment from the whole PCB. Cold mounting is generally used for the fixation of PCB section since PCB section can be stable without the influence of outside pressure, heat and deformation during its grinding process. Epoxy resin for cold mounting is not suitable for quick observation of PCB section since the reaction of epoxy monomer needs long curing time (more than $12 \mathrm{~h}$ ) under the presence of organic amine catalyst. Thus, other polymer system should be found to meet the requirement of quick evaluation of PCB section. Methacrylate monomer could exhibit short copolymerization time at room temperature so.

In this paper, poly (methyl methacrylate)/poly (glycidyl methacrylate) (PMMA/PGMA) blend was prepared for the application of the fixation of PCB section. The performance of PMMA/PGMA blend was investigated to examine its compatibility of cold mounting for PCB-section observation. 


\section{Experimental}

\section{Materials}

MMA and GMA were purchased from Chengdu Kelong Chemical Engineering Co. and RYOJI Co., respectively. PMMA powder was supplied by Arkema Co.

\section{Preparation of PMMA/PGMA Blend}

MMA and GMA were to form a mixed solution. Thereafter, PMMA powder was added into the mixed solution of methyl methacrylate (MMA) and glycidyl methacrylate (GMA).The volume fraction of PMMA powder and MMA/GMA solution was 2:1.Above mixture was cured to obtain PMMA/PGMA blend at room temperature.

\section{Characterization of PMMA/PGMA Blend}

Fourier-transform infrared (FT-IR) spectroscopy was used to determine the chemical composition of MMA, GMA and PMMA/PGMA blend, respectively. Curing time of the mixture was estimated in the silicone mould. Volume shrinkage of PMMA/PGMA blend was examined according to the standard of GB13354-92. Bending strength and tensile strength of PMMA/PGMA blend were measured according to the standards of GB/T 1040-92 and GB/T 9341-2000, respectively. Bonding strength of PMMA/PGMA blend was tested according to the standard of GB6328-86 while PMMA/PGMA blend coated on the surface of copper coil at an area of $3 \mathrm{~mm} \times 2 \mathrm{~mm}$.

\section{Results and Discussion}

\section{FTIR Characterization}

Figure 1 showed FT-IR spectra of PMMA, PGMA and PMMA/PGMA blend. $1726 \mathrm{~cm}^{-1}$ and 2900 $\mathrm{cm}^{-1}$ corresponded to the vibration of ester group and $-\mathrm{CH}_{3}$, respectively. Epoxy group of PGMA at $908 \mathrm{~cm}^{-1}$ was found in the FT-IR spectra of PMMA/PGMA blend [3]. On the other hand, the copolymerization reaction only occurred at carbon-carbon double bond between GMA, MMA and PMMA but not the epoxy group of GMA monomer.

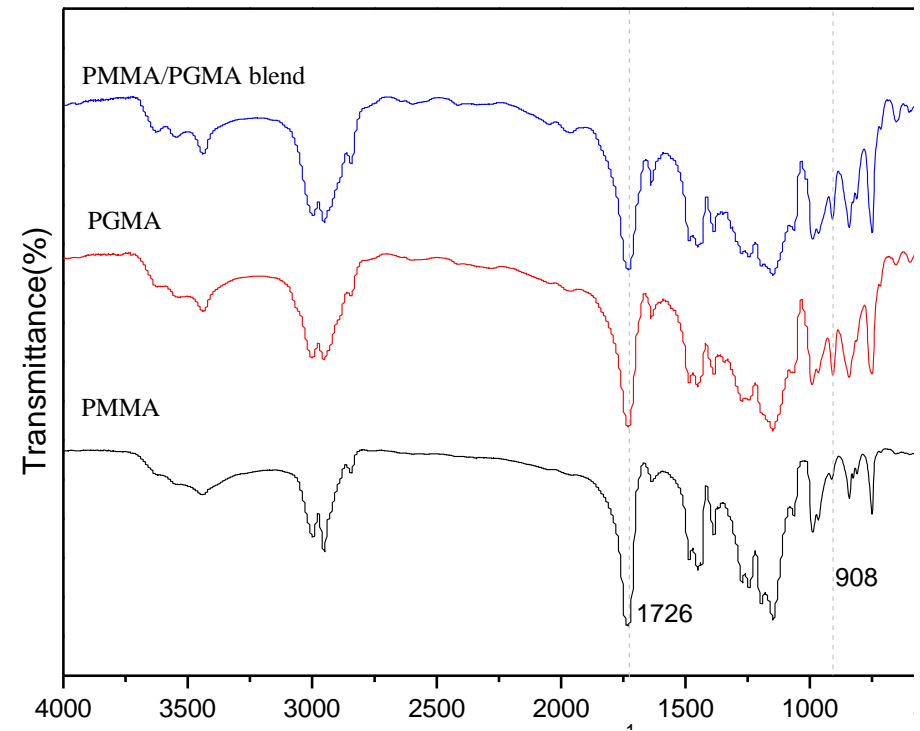

Fig.1 FT-IR spectra of PMMA, PGMA and PMMA/PGMA blend 


\section{Curing Time}

When adding PMMA powder in MMA/GMA solution, copolymerization carried out in carbon-carbon double bond between GMA, MMA and PMMA. The reaction of GMA and MMA or PMMA led to the generation of PGMA while the copolymerization of MMA and PMMA formed PMMA. Thus, PMMA/PGMA blend was finally prepared after curing process. Figure 2 displayed curing time of PMMA powder and MMA/GMA solution. The mixture of PMMA powder and MMA solution resulted in curing time of 33 min while curing time of the mixture of PMMA powder and GMA solution reached to $9 \mathrm{~min}$. Polymerization could induce heat release to promote ring opening ring of epoxy group in GMA to speed up curing rate and shorten curing time [4].

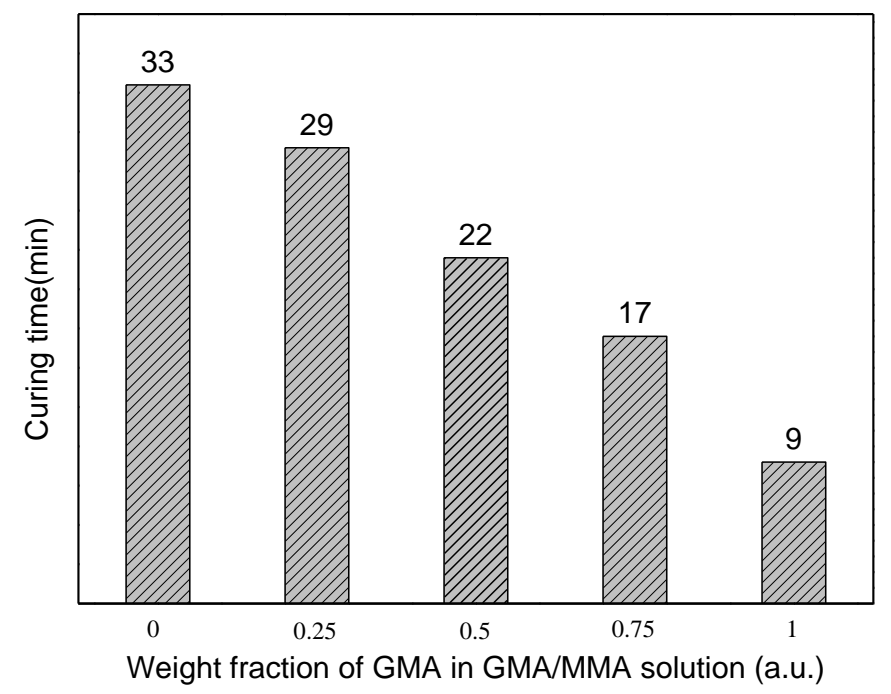

Fig.2 Curing time of the mixture of GMA, MMA and PMMA when adding different weight fraction of GMA in GMA/MMA solution

\section{Volume Shrinkage}

Volume shrinkage of cured process not only could affect the size precision of adhesive but also directly lead to the decrease of the cohesive force. Figure 3 indicated that all the volume shrinkage of PMMA/PGMA blend was less than $10 \%$ when adding different weight fraction of GMA in GMA/MMA solution. Volume shrinkage of PMMA/PGMA blend increased to $9.83 \%$ when the weight fraction of GMA in GMA/MMA solution reached 0.25 . However, when the weight fraction of GMA in GMA/MMA solution was more than 0.25, volume shrinkage of PMMA/PGMA blend tended to decline with the increase of GMA fraction since GMA with bigger functional group and higher molecular weight could result in more space of copolymerization chain for volume shrinkage after curing compared with that of MMA [5]. 


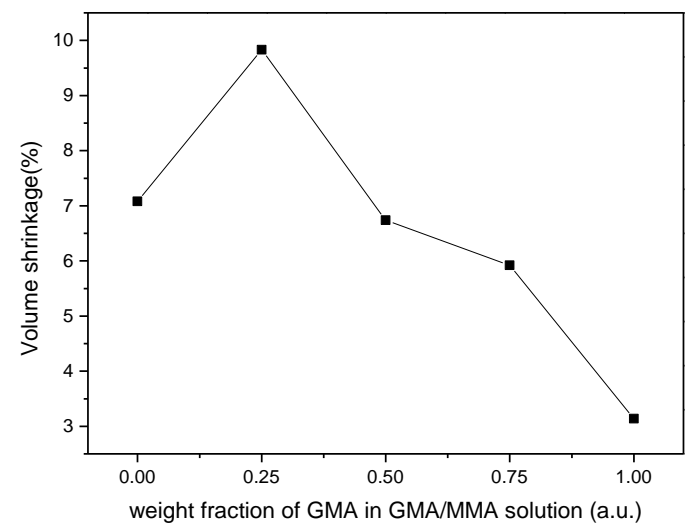

Fig.3 Volume shrinkage of the mixture of GMA, MMA and PMMA when adding different weight fraction of GMA in GMA/MMA solution

\section{Mechanical Properties}

Figure 4 and Figure 5 showed the influence of GMA content on bending strength and tensile strength of PMMA/PGMA blend. Bending strength of PMMA/PGMA blend increased when the weight fraction of GMA in GMA/MMA solution is less than 0.25. Bending strength of PMMA/PGMA blend could reach $49.88 \mathrm{MPa}$ in the condition of 0.25 weight fraction of GMA in GMA/MMA solution. However, when adding more GMA in GMA/MMA solution, bending strength of PMMA/PGMA blend tended to decline. In addition, tensile strength of PMMA/PGMA blend tended to decrease with the increase of GMA in GMA/MMA solution.

Mechanical properties of PMMA/PGMA blend were weakened when adding more GMA in the mixture after curing. Above phenomenon on mechanical properties could be explained as follows: the polar molecule GMA introduced in the blend system could increase the inter-atomic force and reduced the flexibility of molecular chain, thereby reducing the toughness. In addition, adequate content of GMA could form a fine and close interpenetrating networks [6], which strengthened the mechanical properties. However, excessive content of GMA changed the domain size of polymer networks, leading to the weakening of the mechanical properties.

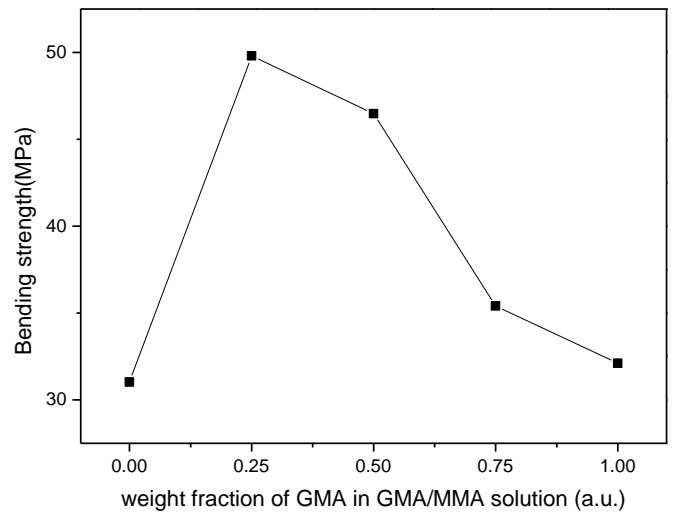

Fig.4 Bending strength of PMMA/PGMA blend when adding different weight fraction of GMA in GMA/MMA solution 


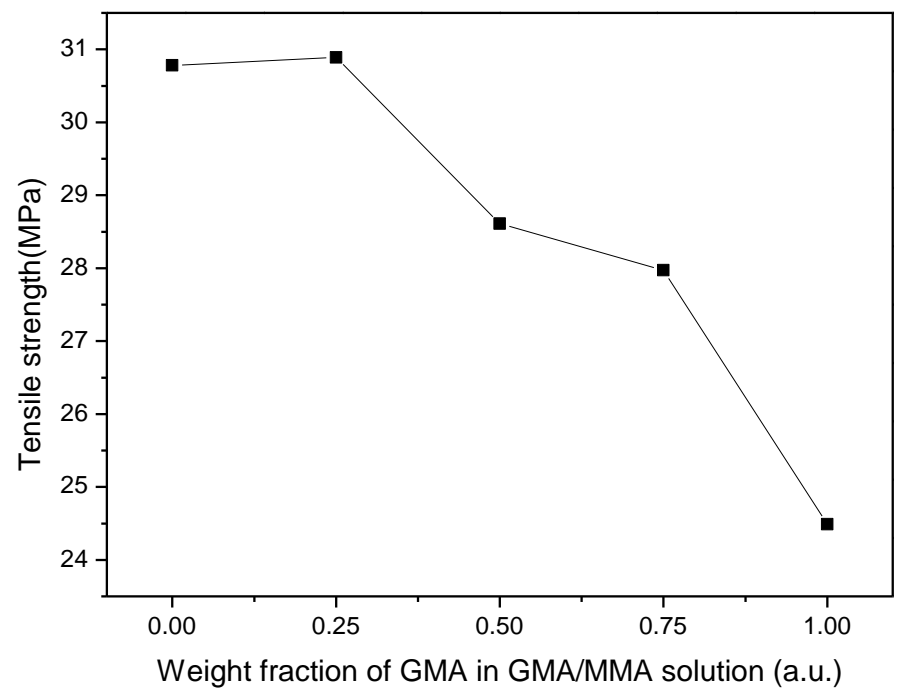

Fig.5 Tensile strength of PMMA/PGMA blend when adding different weight fraction of GMA in GMA/MMA solution

The bonding strength was depicted in Figure 6.Bonding strength of PMMA/PGMA blend decreased with the increase of GMA. The phenomenon was the same as those of the bending strength and tensile strength. Increasing crosslink density could lead to higher the cohesive strength. However, the increase of crosslink density could enhance the viscosity of mixture during curing process so the mixture was hard to be completely cured in a short period of time

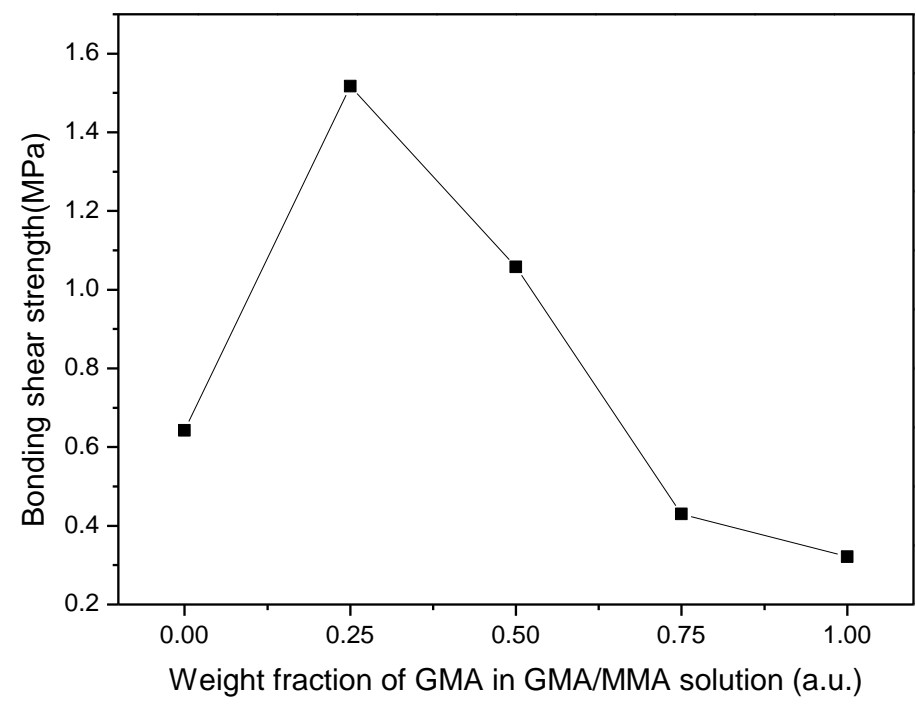

Fig.6 Bonding strength of PMMA/PGMA blend when adding different weight fraction of GMA in GMA/MMA solution

\section{Application of PMMA/PGMA Blend}

From above results, when the weight fraction of GMA in GMA/MMA was 0.25, PMMA/PGMA blend exhibited good performance as curing time of $29 \mathrm{~min}$, volume shrinkage of $9.83 \%$, bending strength of $50 \mathrm{MPa}$, tensile strength of $31 \mathrm{MPa}$ and bonding strength of $1.52 \mathrm{MPa}$. When PCB cross section was put in the mixture of GMA, MMA and PMMA powder (the weight fraction of GMA in 
GMA/MMA was 0.25 and the volume fraction of PMMA powder and MMA/GMA solution was 2:1), No separation of copper and dielectric PCB cross section was found in above metallograph while PMMA/PGMA blend could stably cover PCB cross section after curing process after grinding and polishing, as shown in Figure 7.

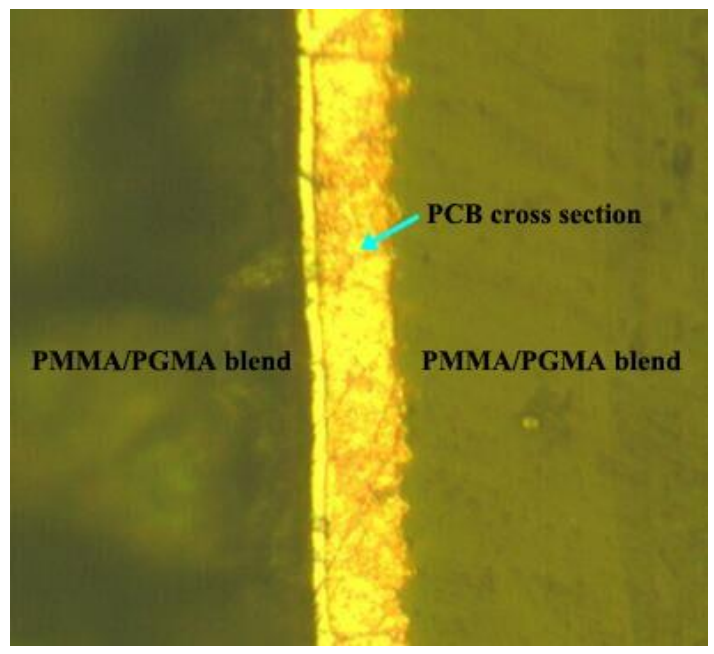

Fig.7 PCB metallographic cross section covered by PMMA/PGMA blend

\section{Conclusion}

When adding 0.25 weight fraction of GMA and MMA into PMMA powder, as-prepared PMMA/PGMA blend could exhibit best performance as short curing time, low volume shrinkage, and high mechanical properties. PMMA/PGMA blend could well cover PCB cross section for stable observation of PCB reliability.

\section{Acknowledgment}

The authors gratefully acknowledge the support of Project of Science and Technology Planning of Guangzhou City China (No. 201604010086), Guangdong Innovative Research Team Program (No.201301C0105324342) and Guangdong Enterprise Key Laboratory Program (No: 2016B030302005).

\section{References:}

[1] Feng Zhou, Shou-xu Wang, Wei He, et al. Study on the Detection of Metallographic Sections in the Process of PCB [J]. Experiment Science and Technology (in Chinese), 2012, 10(6): 18-20.

[2] Qun Zhou. Application of Microsectioning in PCB Production [J].Printed Circuit Information (in Chinese), 2006, 12:60-63

[3] Murat Uygun, Begüm Akduman, Bülent Ergönül, et al. Immobilization of amyloglucosidase onto macroporous cryogels for continuous glucose production from starch [J]. Journal of Biomaterials Science, Polymer Edition, 2015, 26(16): 1112-1125

[4] Witold Brostow, Sidney H, et al. 8-Epoxies, Handbook of Thermoset Plastics (Third Edition) [M].Boston: William Andrew Publishing. 2014: 191-252

[5]Magali Dewaele, Truffer-Boutry Delphine, Jacques Devaux, et al. Volume contraction in photocured dental resins: The shrinkage-conversion relationship revisited [J].Dental Materials, 2006, 22(4):359-365 
[6] Yu-hong Liu, Hui-ming He, Zhong-yi Wang, et al. Study on Dental Plastic IPN Post Composite [J]. Journal of Reinforced Plastics \& Composites, 2010, 29(15): 2684-2690 